\title{
On the analytic continuation of the critical line
}

\author{
Paolo Cea \\ Dipartimento di Fisica dell'Università di Bari and INFN - Sezione di Bari, \\ I-70126 Bari, Italy \\ E-mail: paolo.cea@ba.infn.it

\section{Leonardo Cosmai} \\ INFN - Sezione di Bari, \\ I-70126 Bari, Italy \\ E-mail: leonardo.cosmai@ba.infn.it

\section{Massimo D'Elia} \\ Dipartimento di Fisica dell'Università di Genova and INFN - Sezione di Genova, \\ I-16146 Genova, Italy \\ E-mail: massimo.delia@ge.infn.it
}

\section{Chiara Manneschi}

Dipartimento di Fisica dell'Università di Genova,

I-16146 Genova, Italy

E-mail: chiara.manneschi@ge.infn.it

\section{Alessandro Papa*}

Dipartimento di Fisica, Università della Calabria, and INFN - Gruppo Collegato di Cosenza, I-87036 Rende, Italy

E-mail: papa@cs.infn.it

\begin{abstract}
We perform a numerical study of the systematic effects involved in the determination of the critical line at real baryon chemical potential by analytic continuation from results obtained at imaginary chemical potentials. We present results obtained in a theory free of the sign problem, three-color QCD with finite isospin chemical potential, and comment on general features which could be relevant also to the continuation of the critical line in real QCD at finite baryon density.
\end{abstract}

The XXVII International Symposium on Lattice Field Theory - LAT2009

July 26-31 2009

Peking University, Beijing, China

\footnotetext{
* Speaker.
} 


\section{Introduction}

The study of QCD at non-zero baryon density by numerical simulations on a space-time lattice is plagued by the well-known sign problem: the fermion determinant is complex and the Monte Carlo sampling becomes unfeasible. One of the possibilities to circumvent this problem is to perform Monte Carlo numerical simulations for imaginary values of the baryon chemical potential, where the fermion determinant is real and the sign problem is absent, and to infer the behavior at real chemical potential by analytic continuation. The idea of formulating a theory at imaginary $\mu$ was first suggested in Ref. [1], while the effectiveness of the method of analytic continuation was pushed forward in Ref. [2]. Since then, the method has been extensively applied to QCD [3] 10] and tested in QCD-like theories free of the sign problem [11-16] and in spin models [17, 18]. The state-of-the-art is the following:

- the method is well-founded and works fine within the limitations posed by the presence of non-analyticities and by the periodicity of the theory with imaginary chemical potential [19];

- the analytic continuation of physical observables is improved if ratios of polynomials (or Padé approximants [20]) are used as interpolating functions at imaginary chemical potential [13, 14];

- the analytic continuation of the (pseudo-)critical line on the temperature - chemical potential plane is well-justified, but a careful test in two-color QCD [14] has cast some doubts on its reliability.

In particular, the numerical analysis in two-color QCD of Ref. [14] has shown that, while there is no doubt that an analytic function exists which interpolates numerical data for the pseudo-critical couplings for both imaginary and real $\mu$ across $\mu=0$, determining this function by an interpolation of data at imaginary $\mu$ could be misleading. Indeed, in the case of polynomial interpolations, there is a clear indication in two-color QCD that non-linear terms in $\mu^{2}$ play a relevant role at real $\mu$, but are less visible at imaginary $\mu$, thus calling for an accurate knowledge of the critical line there and, consequently, for very precise numerical data. The above described scenario could well be peculiar to two-color QCD and strongly depend on the choice of parameters of Ref. [14]. Therefore, in this work we perform a systematic study of the analytic continuation of the critical line in another sign-free theory, $\mathrm{SU}(3)$ with a non-zero density of isospin. The dependence on the fermion mass in two-color QCD is considered in a separate contribution [21]. The aim of this study is to single out some general features of the analytic continuation of the critical line and to understand if and to what extent they can apply also to the physically relevant case of QCD.

\section{Analytic continuation of the critical line in three-color QCD at finite isospin chemical potential}

Three-color QCD with a finite density of isospin charge [22] is a theory in which the chemical potential is $\mu$ for half of the fermion species and $-\mu$ for the other half. The partition function, which is even in $\mu$ and depends only on $\mu^{2}$, can be written as follows:

$$
Z(T, \mu)=\int \mathscr{D} U e^{-S_{G}} \operatorname{det} M[\mu] \operatorname{det} M[-\mu],
$$

where the integration is over gauge link variables, $S_{G}$ is the pure gauge action and $M$ the fermion matrix (we adopt a standard staggered discretization). This leads to a real and positive measure, 

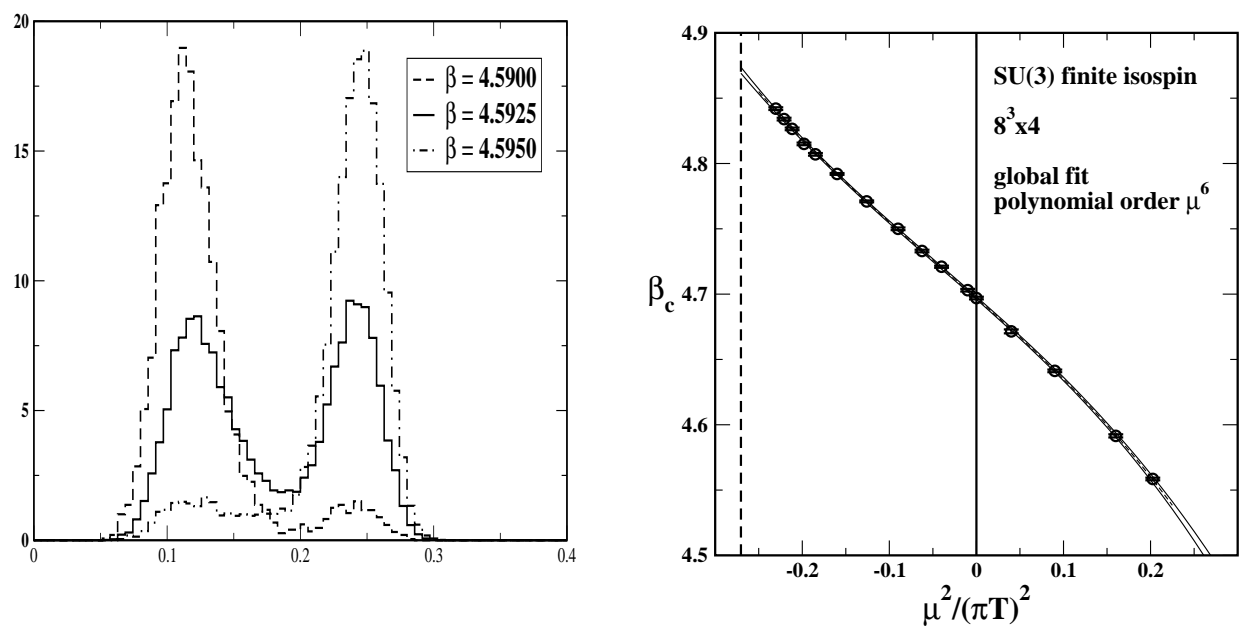

Figure 1: (Left) Distribution of the real part of the Polyakov loop in SU(3) with finite isospin density on a $8^{3} \times 4$ lattice with $a m=0.1$ at $\mu^{2} /(\pi T)^{2}=0.16$ and for three $\beta$ values around the transition. (Right) Critical couplings obtained in $\mathrm{SU}(3)$ with finite isospin density on a $8^{3} \times 4$ lattice with $a m=0.1$, together with a polynomial fit of order $\mu^{6}$ to all data.

because of the property $\operatorname{det} M[-\mu]=(\operatorname{det} M[\mu])^{*}$, and therefore to a theory free of the sign problem. This theory is obviously closer to real QCD than two-color QCD, being yet unphysical, since it implies a zero baryon density, while in Nature a non-zero isospin density is always accompanied by a non-zero baryon density; moreover the isospin charge is not a conserved number in the real world. Nevertheless, for our purposes this theory is very convenient since it provides us with another theoretical laboratory for the method of analytic continuation.

Similarly to SU(2) with finite baryon density, at imaginary values of the chemical potential $\mu$ the theory exhibits RW-like transition lines, the first RW sector being given by the strip $-(0.5)^{2} \lesssim$ $\mu^{2} /(\pi T)^{2} \leq 0$ (we refer to Ref. [23] for a detailed discussion of the QCD phase diagram in presence of an imaginary isospin chemical potential).

In our numerical analysis, we consider finite isospin SU(3) with $N_{f}=8$ degenerate staggered fermions of mass $a m=0.1$ on a $8^{3} \times 4$ lattice. The critical line in the temperature - chemical potential plane is a line of (strong) first order transitions, over all the investigated range of $\mu^{2}$ values, $-0.2304 \leq \mu^{2} /(\pi T)^{2} \leq 0.2025$. This is one of the reasons for working on a small volume (tunneling between the different phases would have been sampled with much more difficulty on a larger volume) and clearly emerges from the distribution on the thermal equilibrium ensemble of the values of observables like the (real part of) the Polyakov loop, the chiral condensate, the plaquette across the transition (see, for example, Fig. 1(left)). Typical statistics have been around $10 \mathrm{~K}$ trajectories of $1 \mathrm{MD}$ unit for each run, growing up to $100 \mathrm{~K}$ trajectories for 2-3 $\beta$ values around $\beta_{c}\left(\mu^{2}\right)$, for each $\mu^{2}$, in order to correctly sample the critical behavior at the transition. The critical $\beta\left(\mu^{2}\right)$ is determined as the point where the two peaks have equal height and, in all the cases considered, this point turned out to be the same for all the adopted observables.

\subsection{Results for the critical line at finite isospin}

The general strategy is the following: after determining, for a set of $\mu^{2}$ values, the critical 

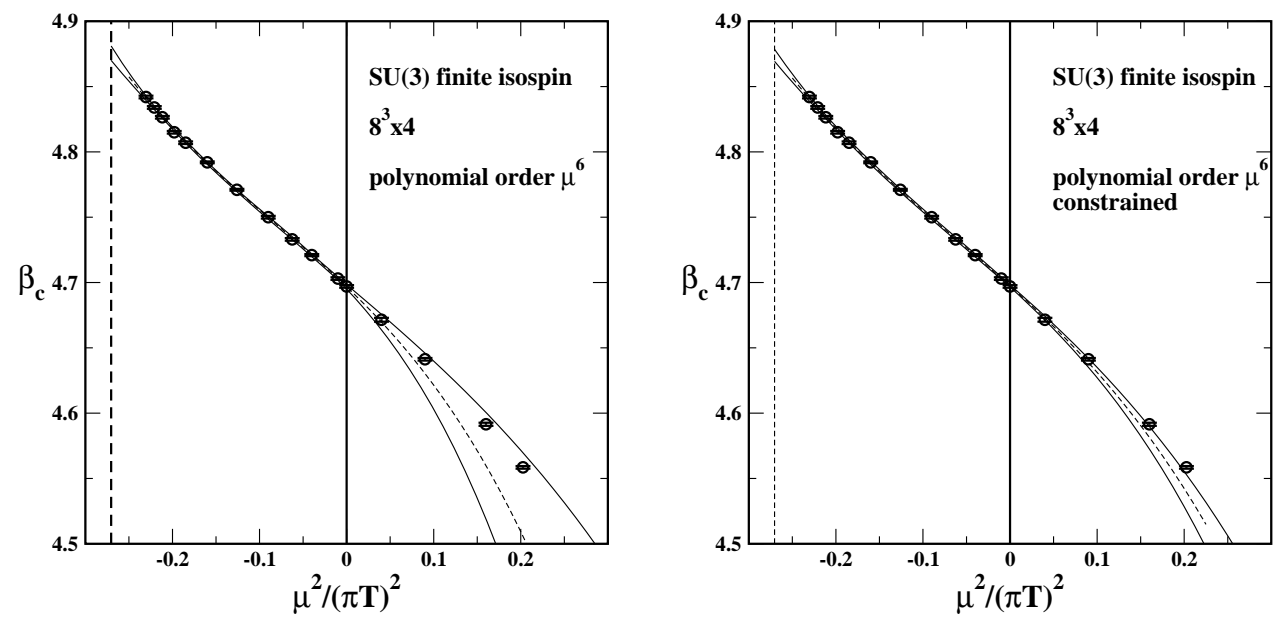

Figure 2: (Left) Critical couplings obtained in SU(3) with finite isospin density on a $8^{3} \times 4$ lattice with $a m=0.1$, together with a polynomial fit of order $\mu^{6}$ to data with $\mu^{2} \leq 0$. (Right) The same with a polynomial of order $\mu^{6}$ with constrained quadratic term.

couplings $\beta_{c}\left(\mu^{2}\right)$, the critical line is guessed by interpolating the values of $\beta_{c}\left(\mu^{2}\right)$ for $\mu^{2} \leq 0$ only. The validity of the interpolation is evaluated by comparing its analytic continuation to the region $\mu^{2}>0$ with the direct determinations of the critical coupling in this region ${ }^{1}$.

We observe from the very beginning that data for $\beta_{c}\left(\mu^{2}\right)$ for both $\mu^{2} \leq 0$ and $\mu^{2}>0$ can be globally fitted by an analytic function (a polynomial of third order in $\mu^{2} /(\pi T)^{2}$ nicely works). Fig. 11(right) shows how the fit compares with data. The question is if there are interpolations of the critical couplings at $\mu^{2} \leq 0$ only, that, when continued to $\mu^{2}>0$, agree with the critical couplings directly determined in the latter region.

We have tried several kind of interpolations of the critical couplings at $\mu^{2} \leq 0$. At first, we have considered interpolations with polynomials up to order $\mu^{10}$. We can see that data at $\mu^{2} \leq 0$ are precise enough to be sensitive to terms beyond the order $\mu^{2}$; indeed, a good $\chi^{2} /$ d.o.f. is not achieved before including terms up to the order $\mu^{6}$, in agreement with the outcome of the global fit discussed above. The extrapolation to $\mu^{2}>0$ for the polynomial of order $\mu^{6}$ is shown in Fig. 2(left); it agrees with direct determinations of $\beta_{c}\left(\mu^{2}\right)$, within the $95 \%$ CL band.

Then, we have considered interpolations with ratios of polynomials of order up to $\mu^{6}$. In all but one cases we got good fits to the data at $\mu^{2} \leq 0$, but only two extrapolations to $\mu^{2}>0$ compare well with numerical data in that region: the ratio of a 4th to 6th order polynomial and the ratio of a 6th to 4th order polynomial [23]. It is interesting to observe that the two interpolations which "work" have in common the number of parameters.

Both kinds of fits considered so far have evidenced that the role of terms of order larger than $\mu^{2}$ cannot be neglected. Since the data more sensitive to these terms are those farther from $\mu^{2}=0$, while data closer to $\mu^{2}=0$ should "feel" only the $\mu^{2}$ term in a polynomial interpolation, we performed a fit with a polynomial of the form $a_{0}+a_{1} \mu^{2} /(\pi T)^{2}$ in a small region $\mu^{2} \lesssim 0$ and fixed the value of the parameter $a_{1}$ (see Ref. [23] for details). Then, we kept $a_{1}$ fixed and repeated the fit

\footnotetext{
${ }^{1}$ We refer to Ref. [23] for all the determinations of the critical couplings in the finite isospin SU(3) theory on a $8^{3} \times 4$ lattice with fermion mass $a m=0.1$ and for the parameters of all the fits presented below.
} 

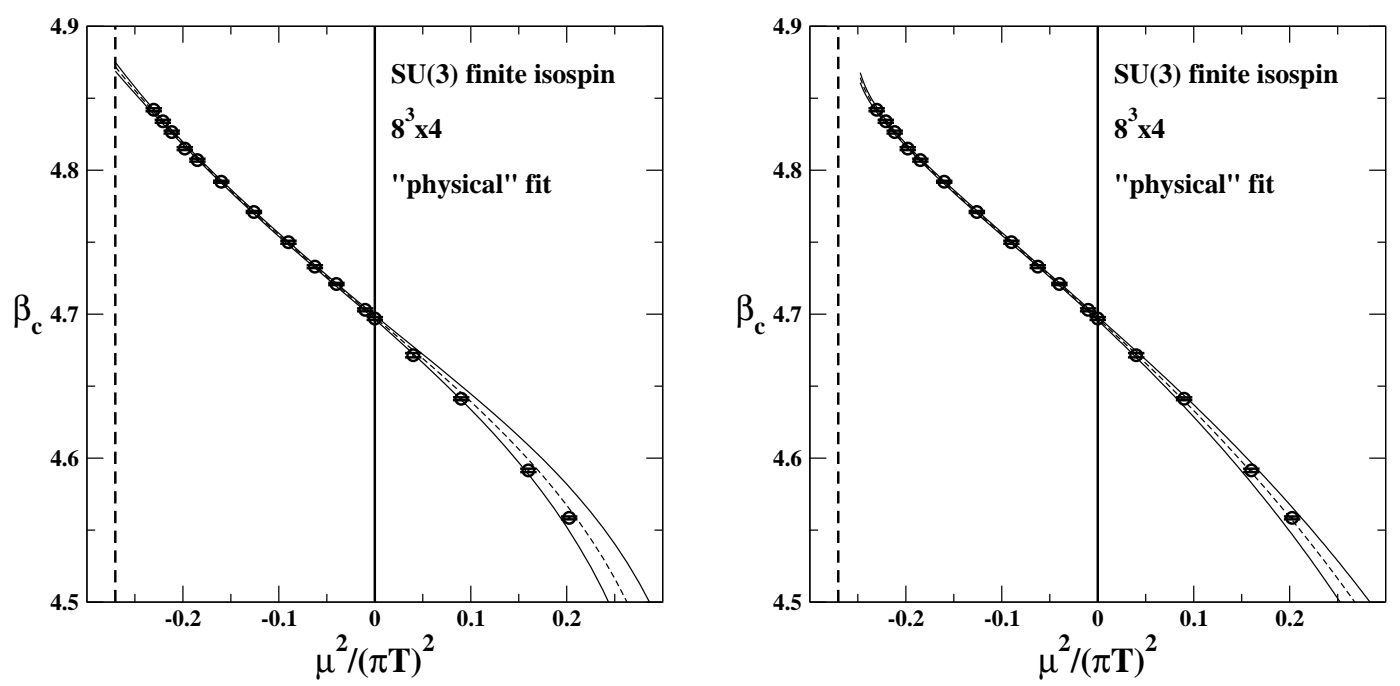

Figure 3: Values of $\beta_{c}\left(\mu^{2}\right)$ in $\mathrm{SU}(3)$ with finite isospin density on a $8^{3} \times 4$ lattice with am=0.1, together with the fit to data with $\mu^{2} \leq 0$ according to the fit functions (2.3)(left) and (2.4)(right).

on all available data at $\mu^{2} \leq 0$ with a polynomial of the form $a_{0}+a_{1} \mu^{2} /(\pi T)^{2}+a_{2} \mu^{4} /(\pi T)^{4}+$ $a_{3} \mu^{6} /(\pi T)^{6}$. The resulting interpolation and its extrapolation to the region $\mu^{2}>0$ are shown in Fig. 2 (right). The comparison with critical couplings at $\mu^{2}>0$ is good and the $95 \%$ CL band is narrower than in the unconstrained $\mu^{6}$-polynomial fit (see Fig. Q $($ (right)), meaning that this procedure leads to increased predictivity for the method of analytic continuation.

At last, we have attempted the fit strategy to write the interpolating function in physical units and to deduce from it the functional dependence of $\beta_{c}$ on $\mu^{2}$, after establishing a suitable correspondence between physical and lattice units. The natural, dimensionless variables of our theory are $T / T_{c}(0)$, where $T_{c}(0)$ is the critical temperature at zero chemical potential, and $\mu /(\pi T)$. The question that we want to answer is if fitting directly the dependence of $T / T_{c}(0)$ on $\mu /(\pi T)$ may lead to increased predictivity for analytic continuation. We shall name this kind of fits as "physical" fits. While $\mu /(\pi T)$ is one of the dimensionless variables used in our simulations, $T / T_{c}(0)$ is not and must be deduced from the relation $T=1 /\left(N_{t} a(\beta)\right)$, where $N_{t}$ is the number of lattice sites in the temporal direction and $a(\beta)$ is the lattice spacing at a given $\beta^{2}$. Since our determinations for $\beta_{c}$ range between $\simeq 4.5585$ and $\simeq 4.842$, it can make sense to use for $a(\beta)$ the perturbative 2-loop expression with $N_{c}=3$ and $N_{f}=8$.

We have tried several different fitting functions and report two cases which work particularly well. The first is given by the following 3-parameter function:

$$
\left[\frac{T_{c}(\mu)}{T_{c}(0)}\right]^{2}=\frac{1+B \mu^{2} /\left(\pi T_{c}(\mu)\right)^{2}}{1+A \mu^{4} /\left(\pi T_{c}(\mu)\right)^{4}}
$$

\footnotetext{
${ }^{2}$ Strictly speaking the lattice spacing depends also on the bare quark mass, which in our runs slightly changes as we change $\beta$ since we fix am. However in the following evaluation, which is only based on the perturbative 2-loop $\beta$-function, we shall neglect such dependence.
} 
leading to the following implicit relation between $\beta_{c}$ and $\mu^{2}$ :

$$
\left.a\left(\beta_{c}\left(\mu^{2}\right)\right)^{2}\right|_{2-\text { loop }}=\left.a\left(\beta_{c}(0)\right)^{2}\right|_{2-\text { loop }} \frac{1+A \mu^{4} /\left(\pi T_{c}(\mu)\right)^{4}}{1+B \mu^{2} /\left(\pi T_{c}(\mu)\right)^{2}} .
$$

In Fig. 3 (left) we compare to data the fit with the function (2.3): one can see that the extrapolation to the region $\mu^{2}>0$ behaves very well. The values of the fit parameters are $\beta_{c}(0)=4.6977(13)$, $A=-3.25(26)$ and $B=-2.62(12)$, with $\chi^{2} /$ d.o.f. $=1.33$.

As an alternative function for the shape of the critical line, we have tried also the following

$$
\frac{T_{c}(\mu)}{T_{c}(0)}= \begin{cases}A+(1-A)\left[\cos \left(\frac{\mu}{T}\right)\right]^{B}, & \mu^{2} \leq 0 \\ A+(1-A)\left[\cosh \left(\frac{\mu}{T}\right)\right]^{B}, & \mu^{2}>0\end{cases}
$$

which explicitly encodes the expected periodicity of the partition function for imaginary $\mu$. The fit to data at imaginary $\mu$ is very good and its extrapolation to the real chemical potential side compares impressively well with data (see Fig. 3)(right). The resulting fit parameters are $\beta_{c}(0)=4.6969(12)$, $A=1.508(15)$ and $B=0.560(32)$, with $\chi^{2} /$ d.o.f. $=0.39$. This function is a good candidate to parameterize the critical line for small values of $\mu / T$.

In both cases, Eq. (2.3) and Eq.(2.4), the "physical" fit worked very well and with a reduced number of parameters with respect to our previous fits, leading to increased predictivity and consistency with data at real chemical potentials. One can easily check that the adopted functions are not appropriate for a continuation of the critical line down to the $T=0$ axis, but this is not the aim of our study, since such extrapolation would be questionable anyway.

\section{Conclusions}

In this work we have presented results concerning the analytic continuation of the critical line in QCD with a finite density of isospin charge. We have detected some features and developed some strategies, which could apply and be useful for real QCD at finite baryon density. Let us briefly summarize them.

- Non-linear terms in the dependence of the pseudocritical coupling $\beta_{c}$ on $\mu^{2}$ in general cannot be neglected. A polynomial of order $\mu^{6}$ seems to be sufficient in all explored cases.

- The coefficients of the linear and non-linear terms in $\mu^{2}$ in a Taylor expansion of $\beta_{c}\left(\mu^{2}\right)$ are all negative. That often implies subtle cancellations of non-linear terms at imaginary chemical potentials $\left(\mu^{2}<0\right)$ in the region available for analytic continuation (first RW sector). The detection of such terms, from simulations at $\mu^{2}<0$ only, may be difficult and requires an extremely high accuracy. As a matter of fact, the simple use of a sixth order polynomial to fit data at imaginary $\mu$ leads to poor predictivity, which is slightly improved if ratio of polynomials are used instead.

- An increased predictivity is achieved if the linear term in $\mu^{2}$ is fixed from data at small values of $\mu^{2}$ only.

- We have proposed a new, alternative ansatz to parameterize the critical line directly in physical units in the $T, \mu$ plane (instead than in the $\beta, \mu$ plane) and given two explicit realizations. This "physical" ansatz provides a very good description of the critical line, moreover with a reduced number of parameters, and leads to an increased predictivity, comparable to that achieved by the "constrained" fit. 


\section{References}

[1] M.G. Alford, A. Kapustin, and F. Wilczek, Phys. Rev. D59 (1999) 054502 [hep-lat/980 039 ].

[2] M.-P. Lombardo, Nucl. Phys. Proc. Suppl. 83 (2000) 375 [hep-lat/9908006].

[3] Ph. de Forcrand and O. Philipsen, Nucl. Phys. B642 (2002) 290 [hep-lat/0205016]; Nucl. Phys. B673 (2003) 170 [hep-lat/0307020].

[4] M. D'Elia and M.P. Lombardo, Phys. Rev. D67 (2003) 014505 [hep-lat / 0209146 ]; Phys. Rev. D70 (2004) 074509 [hep-lat / 0406012 ].

[5] V. Azcoiti, G. Di Carlo, A. Galante and V. Laliena, Nucl. Phys. B723 (2005) 77 [hep-lat/0503010].

[6] H.S. Chen, X.Q. Luo, Phys. Rev. D72 (2005) 034504 [hep-lat / 0411023 ].

[7] Ph. de Forcrand and O. Philipsen, JHEP 0701 (2007) 077 [hep-lat / 0607017 ]; POS (LATTICE2007)178 [arXiv:0711.0262].

[8] L.K. Wu, X.Q. Luo and H.S. Chen, Phys. Rev. D76 (2007) 034505 [hep-lat/ 0611035 ].

[9] M. D’Elia, F. Di Renzo, M.P. Lombardo, Phys. Rev. D76 (2007) 114509 [arXiv: 0705.3814 ].

[10] M. D’Elia and F. Sanfilippo, Phys. Rev. D80 (2009) 014502 [arXiv: 0904 . 1400]; [arXiv:0909.0254].

[11] A. Hart, M. Laine, and O. Philipsen, Phys. Lett. B505 (2001) 141 [hep-lat/ 0010008 ].

[12] P. Giudice and A. Papa, Phys. Rev. D69 (2004) 094509 [hep-lat / 0401024 ]; Nucl. Phys. Proc. Suppl. 140 (2005) 529 [hep-lat/ 0409022$].$

[13] P. Cea, L. Cosmai, M. D'Elia and A. Papa, JHEP 0702 (2007) 066 [hep-lat / 0612018 ]; PoS (LAT2006) 143 [hep-lat/0610088].

[14] P. Cea, L. Cosmai, M. D’Elia and A. Papa, Phys. Rev. D77 (2008) 051501 [arXiv: 0712 . 3755]; PoS (LATTICE 2007) 214 [arXiv:0710.2068].

[15] S. Conradi, M. D’Elia, Phys. Rev. D76 (2007) 074501 [arXiv: 0707 . 1987].

[16] Y. Shinno and H. Yoneyama, arXiv:0903.0922.

[17] S. Kim, P. de Forcrand, S. Kratochvila, and T. Takaishi, POS (LAT2005) 166 [hep-lat/0510069].

[18] F. Karbstein, M. Thies, Phys. Rev. D75 (2007) 025003 [hep-th/ 0610243 ].

[19] A. Roberge, N. Weiss, Nucl. Phys. 275 (1986) 734.

[20] M.-P. Lombardo, PoS (LAT2005) 168 [hep-lat/0509181].

[21] P. Cea, L. Cosmai, M. D’Elia and A. Papa, PoS (LAT2009) 192.

[22] D.T. Son and M.A. Stephanov, Phys. Rev. Lett. 86 (2001) 592 [hep-ph/ 0005225 ]; J.B. Kogut and D.K. Sinclair, Phys. Rev. D66 (2002) 034505 [hep-lat/0202028].

[23] P. Cea, L. Cosmai, M. D’Elia, C. Manneschi and A. Papa, Phys. Rev. D80 (2009) 034501 [arXiv:0905.1292]. 